\title{
An Epidemic Grid Model to Address the Spread of Covid-19: A Comparison between Italy, Germany and France
}

\author{
Maria Teresa Signes-Pont *, José Juan Cortés-Plana and Higinio Mora-Mora \\ Department of Computer Science and Technology, University of Alicante, Carr. de San Vicente del Raspeig, s/n, \\ 03690 San Vicente del Raspeig, Alicante, Spain; jj.cortes@ua.es (J.J.C.-P.); hmora@dtic.ua.es (H.M.-M.) \\ * Correspondence: teresa@dtic.ua.es
}

Citation: Signes-Pont, M.T.;

Cortés-Plana, J.J.; Mora-Mora, H. An Epidemic Grid Model to Address the Spread of Covid-19: A Comparison between Italy, Germany and France. Math. Comput. Appl. 2021, 26, 14. https://doi.org/10.3390/ mca26010014

Received: 5 January 2021

Accepted: 2 February 2021

Published: 8 February 2021

Publisher's Note: MDPI stays neutral with regard to jurisdictional claims in published maps and institutional affiliations.

Copyright: (c) 2021 by the authors. Licensee MDPI, Basel, Switzerland. This article is an open access article distributed under the terms and conditions of the Creative Commons Attribution (CC BY) license (https:/ / creativecommons.org/licenses/by/ $4.0 /)$.

\begin{abstract}
This paper presents a discrete compartmental Susceptible-Exposed-Infected-Recovered/ Dead (SEIR/D) model to address the expansion of Covid-19. This model is based on a grid. As time passes, the status of the cells updates by means of binary rules following a neighborhood and a delay pattern. This model has already been analyzed in previous works and successfully compared with the corresponding continuous models solved by ordinary differential equations (ODE), with the intention of finding the homologous parameters between both approaches. Thus, it has been possible to prove that the combination neighborhood-update rule is responsible for the rate of expansion and recovering/death of the disease. The delays (between Susceptible and Asymptomatic, Asymptomatic and Infected, Infected and Recovered/Dead) may have a crucial impact on both height and timing of the peak of Infected and the Recovery/Death rate. This theoretical model has been successfully tested in the case of the dissemination of information through mobile social networks and in the case of plant pests.
\end{abstract}

Keywords: Covid-19; computational modelling; space time framework; multigrid implementation; update binary rules; Von Neumann and Moore neighborhoods

\section{Introduction}

Since December 2019, the world has been facing the most serious pandemic since the Spanish flu pandemic of 1918. World Health Organization (WHO) declared the pandemic on 11 March 2020. The outbreak has caused nearly 103,000,000 infections and more than 2,200,000 deaths as of the time of this publication. In response to this threat, all governments have ordered different degrees of containment measures, such as school and workplace closures, travel bans and quarantines, and have launched actions including social distancing, mandatory mask wearing, hand hygiene and viral or antibody testing campaigns as well as contact tracing using cell phone tracking.

Many researchers have dedicated their efforts to coping with the dynamics of the spread of the disease; that is, they have provided insight into the underlying mechanisms of the virus propagation in order to guide the planning of the public health policies to ensure success in the fight against COVID-19.

Our work aims to study the spread of the virus. Our approach is based on the compartmental model paradigm and we use a grid to cope with the process of expansion. Three parameters define the dynamics of the expansion. A neighborhood fixes the cells that may be infected by an initial infected cell, an update rule implements the changes of the value of the neighbor cell, when it becomes Infected or Recovered. Finally, the time it takes for a cell to transition from one state to another fixes the delay between compartments.

Following the introduction, Section 2 analyzes the related works in which researchers approach Covid-19 modelling using compartmental models and presents in detail our method. Section 3 provides a first insight into the data of confirmed cases and deaths in three European countries, Italy, Germany and France and applies our method as a suitable 
tool to approximate the trends of the pandemic's expansion. Finally, Section 4 presents concluding remarks and some future research lines.

\section{Materials and Methods}

\subsection{Related Work}

In the following, we review some publications in which researchers approach Covid19 using compartmental models. Reference [1] presents a summary of the SusceptibleInfected-Recovered (SIR) model and its variants SEIR (with an Exposed E compartment) and Susceptible-Unquarantined infected-Quarantined infected-Confirmed infected (SUQC) models, in order to highlight the relationship between the health measures to curb the pandemic and the mathematics behind them. Although this work has no real research impact, it is very useful as a link between computer scientists and physicians. In [2] the authors analyze the SIR and SEIR models with the complementary Quarantine, Lockdown and Vaccine compartments. They also simulate the SIR model to explore the dynamics of coronavirus in Ghana based on available data up until 9 April 2020. Reference [3] divides the population into the following states: susceptible subjects (S), have close contacts (C, those exposed to infected subjects / pathogen but not necessarily infected), latent (E, infected and infectious but asymptomatic), infected (I; and symptomatic), recovered (V), and dead (D). This four-compartment model is applied to evaluate the epidemiological data and hospital burden in Italy, the UK, and the US. The control measures are identified as the key drivers for the observed epidemiological data through sensitivity analyses and provide a framework for major pandemics in the future. In [4], the authors introduce random sampling scheme that enables a stochastic extension of the SIR model to provide the ability to quantify uncertainty in both estimation and prediction, in connection to sampling variability. This added uncertainty is very useful because the model not only generates an average estimation or prediction, it also presents the best and worst possible scenarios. This is critical for policies that enable more robust and secure management of epidemics. In reference [5] the authors generalize the simple 3-compartment SIR model to the SLIAR model, by considering L (latency) and A (asymptomatic) compartments. This provides a reasonable approximation to the details of progression of the pandemic with a minimal number of parameters. The novelty is the further incorporation of Erlang distributions of the time of sojourn in some of the important compartments. In [6] the model is then described by a dynamical system enclosing a closed population with Susceptible (S), confirmed Infected (I), Asymptomatic (A), Quarantined (Q), Recovered (R) and Died (D), people. The authors conclude that the value of the peak, and thus the impact on the health care system, depends critically on the proportion of cases that are asymptomatic. In Spain, many research teams also present different approaches. At the R. Margaleff Institute of Research (University of Alicante), Dr. C. Bordehore leads a multidisciplinary research team which studies the spread of Covid-19. He follows an open SEIR model which can include different realistic scenarios to provide an estimate of the impact of each parameter on the spread. The model is currently in an update phase that aims to define R0 with more accuracy [7]. The Modelling Uncertainty Quantification group (MUNQU) at the Polytechnic University of Valencia has presented an epidemic model in order to provide a daily prediction of the expansion of Covid-19 which is available at https: / / covid19.webs.upv.es / (accessed on 9 March 2021). The model is based on the SIR paradigm, with 9 compartments: SusceptibleQuarantine-Latent-Infected-Hospitalized-ICU (intensive care unit)-Hospitalized post ICU-Recovered-Dead. The system is solved by a set of differential ordinary equations (ODE) [8]. All these references highlight compartmental models which are suitable for approaching the expansion of infectious diseases

\subsection{Method}

Our model is based on a discrete time space approach that follows a typical epidemic paradigm (SIR, SEIR ... ). We substitute the ODE for a framework implemented by a grid in which an "infected" cell spreads the disease to its neighbors when time passes. Different 
types of neighborhood are proposed (Von Neumann, Moore and "L" or Chess horse jump), along with a local binary update rule, which defines the result of the interaction between neighbors in terms of contagion. Figure 1 shows the initial infected cell at $\mathrm{T}=0$ (red) and the spread of the disease at $\mathrm{T}=1$ and $\mathrm{T}=2$ for different neighborhood types. To ease the explanation, the number inside the infected cells stands for the time (generation) they become infected. The white cells are susceptible.
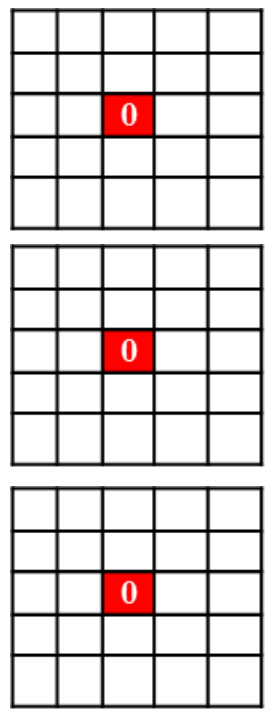
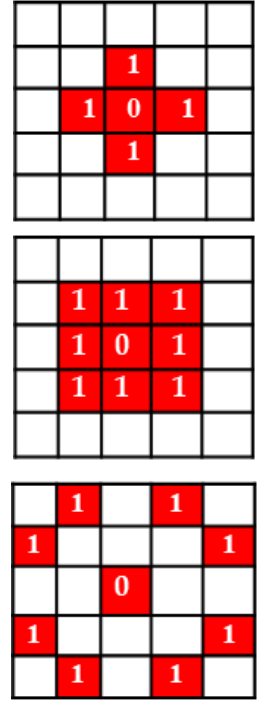
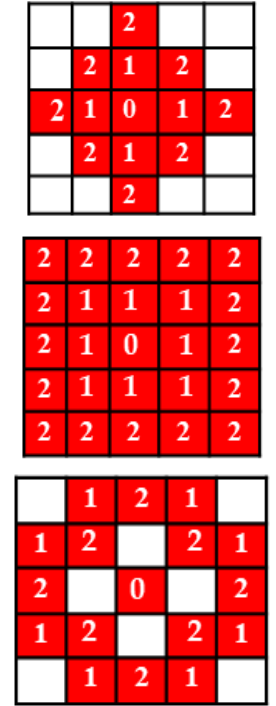

Von Neumann

Neighborhood

Moore

Neighborhood

Chess horse jump or "L"

Neighborhood

Von Neumann neighborhood: The neighbors of a cell are its four adjacent cells.

Moore neighborhood: The neighbors of a cell are the eight cells that surround it.

" $\mathrm{L}$ " or Chess horse jump neighborhood: The neighbors of a cell are the cells that follow the chess horse jump scheme (they move making the shape of letter $\mathrm{L}$ )

Figure 1. The grid implementation for three different neighborhood types, at $\mathrm{T}=0,1$ and 2 .

This discrete grid model has been deeply analyzed and compared with the continuous models solved by ordinary differential equations (ODE), with the intention of finding the homologous parameters between both approaches [9-15]. Three parameters are crucial for the characterization of our proposal: neighborhoods, delays and update rules. Neighborhoods allow different speeds of propagation. They also determine the height and timing of the peak of the outbreak. The neighborhoods play the role of parameters $\beta$ infection rate), and $\gamma$ (recovery rate) in ODE.

The binary update rules $R_{m}$ define the transition between the different stages of a cell. They are defined by two-input tables, as follows:

\begin{tabular}{|l|l|l|}
\cline { 2 - 3 } \multicolumn{1}{c|}{$*$} & $\mathbf{0}$ & $\mathbf{1}$ \\
\hline $\mathbf{0}$ & a3 & a1 \\
\hline $\mathbf{1}$ & a2 & a0 \\
\hline
\end{tabular}

$\forall \mathrm{i}, \mathrm{ai}_{\mathrm{i}} \in\{0,1\}$

There are 16 different update rules $\mathrm{R}_{\mathrm{m}}$. Each rule is assigned a number, $m$, to easily differentiate it from the others. The number is $m=a_{3} a_{2} a_{1} a_{0}$ (a sequence of four bits).

As an example, for $\mathrm{R}_{7}$, we have $\mathrm{m}=7=\mathrm{a}_{3} \mathrm{a}_{2} \mathrm{a}_{1} \mathrm{a}_{0}=0111$ and the operation defined by the $R_{7}$ table is:

$$
\begin{aligned}
& (0 * 0)=a_{3}=1 \\
& (1 * 0)=a_{2}=1 \\
& (0 * 1)=a_{1}=1 \\
& (1 * 1)=a_{0}=0
\end{aligned}
$$


So, as an example, when the value of two neighbor cells are $(1,0)$ at time $=T, R_{7}$ will update the value of the second cell and, at $T+1$, the value of the cells will be $(1,1)$, since the first cell (1) infects the second (0) and sets it to 1, as shown in Figure 1.

For any neighborhood, the delays in the application of the rules have an impact on the duration of the disease, on the peak of Infected and on the equilibrium values of the number of cells in each stage (Susceptible, Exposed, Infected, Recovered etc.) [16].

Time is counted in generations to cope with different times, according to the dynamics of any particular disease. It is a flexible parameter to facilitate the match between the model and the actual data, since the model is just a template.

In the first three subfigures of Figure 2 the number of Susceptible, Infected and Recovered vs. time (generation) are plotted, in the case of the SIR model and for different neighborhoods. We also present the simulation of the SIR model by ODE for comparison. We observe the similar behavior of the compartments S, I and R in ODE and grid models. We also observe how neighborhoods allow different speeds of propagation: the peak of the Infected is reached in generation 6 for the Von Neumann neighborhood (the slowest) and in generation 3 for the L neighborhood (the fastest). The neighborhood also determines the height of the peak. The highest is for the L neighborhood and the lowest for the Von Neumann neighborhood. These parameters are equivalent to $\beta$ and $\gamma$ in the ODE model.

In the last two subfigures, the delays in the application of the update rules (that is the number of generations a cell remains in a determined group: susceptible, infected, recovered before it moves to the next one...) have an impact on the duration of the disease and on the peak of Infected, for any neighborhood (two figures at the bottom). Only the case of the Von Neumann neighborhood is shown, but the same can be observed for other neighborhoods [16].

Since the single grid model provides a first behavioral approximation of the expansion, a more complex and realistic model is achieved by means of a multigrid implementation, that is, a set of grids in a vertical stack, where each grid depicts the impact of a single circumstance or characteristic on the spread of the disease. As an example, when individual nodes have a behavior that goes beyond what is expected, this alteration cannot be highlighted by means of ODE. Reference [16] presents in chapter 4, pp. 82-117 the impact of human action and decision on the dissemination of information in mobile social networks. The model considers both a normal behavior (an ignorant node becomes spreading and then recovered) and an abnormal one (some ignorant nodes spontaneously recover without spreading, or some spreading nodes spontaneously recover without forwarding the message). A set of two superposed grids can depict these situations using SIR models. The second grid carries out a periodic alteration of the normal behavior of the cells in the main grid. The frequency of alteration of the second grid has a crucial impact. The results prove that a high frequency alteration jeopardizes the normal diffusion speed (nodes become "selfish" because they "decide" not to send information and that corresponds to the actual observations).

The multigrid implementation is a valuable tool to depict minority individual behaviors, which may have an impact on the global dynamics of a population. 

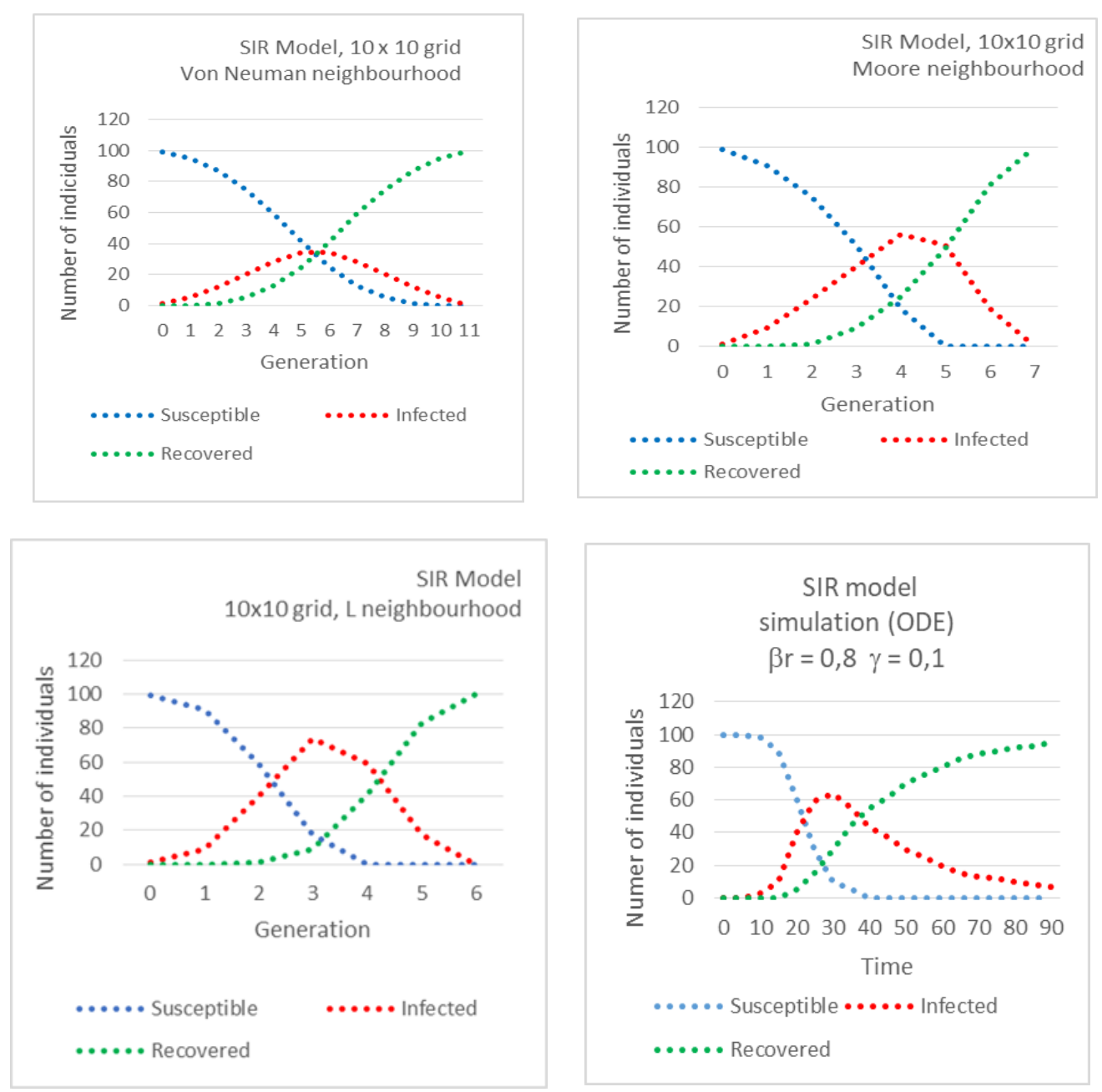

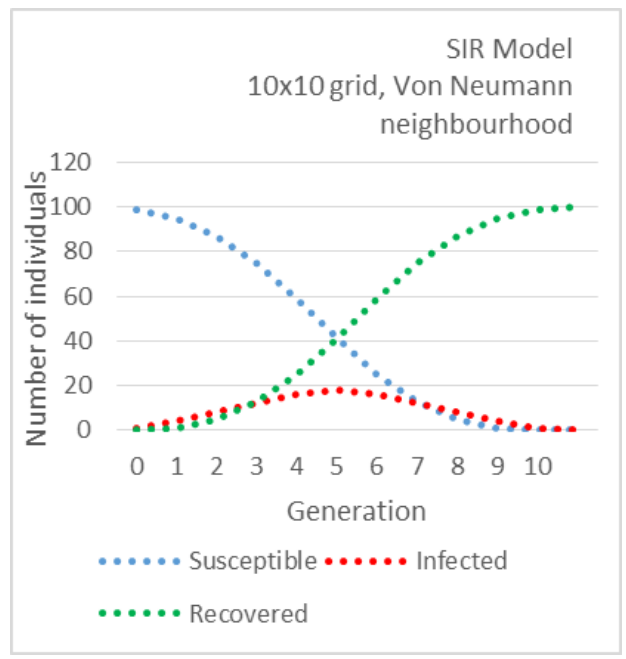

The disease lasts 1 generation

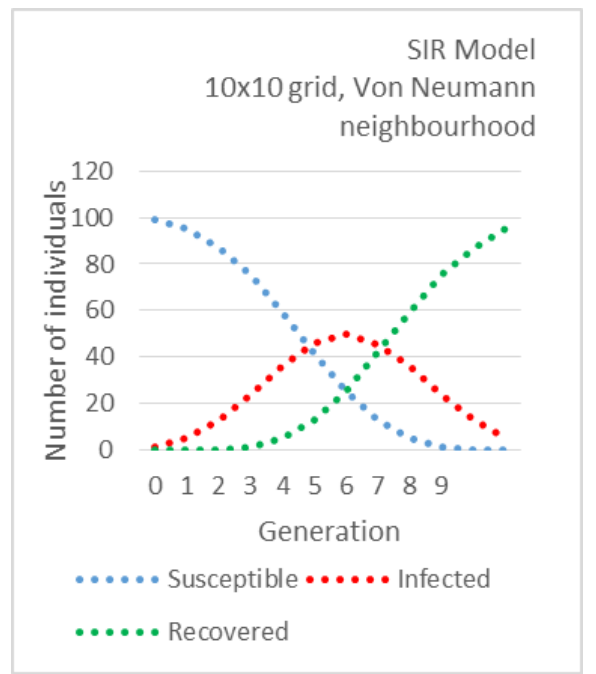

The disease lasts 3 generations

Figure 2. Number of Susceptible, Infected and Recovered vs. time (generation) in the case of an Susceptible-Infected-Recovered (SIR) model, for different neighborhoods. 


\section{Results}

\subsection{The Expansion of Covid-19 in three European Countries}

We must have a first insight into the data of confirmed cases and deaths. consider the pandemic in three European countries: Italy, Germany and France, between 15 February and 22 July 2020. Data are collected from Worldometer for Coronavirus Disease [17] and plotted in graphs. See Figure 3a,c,e.

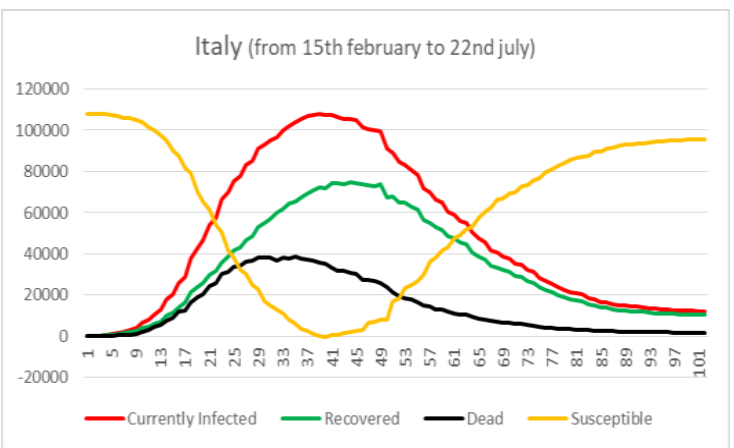

(a)

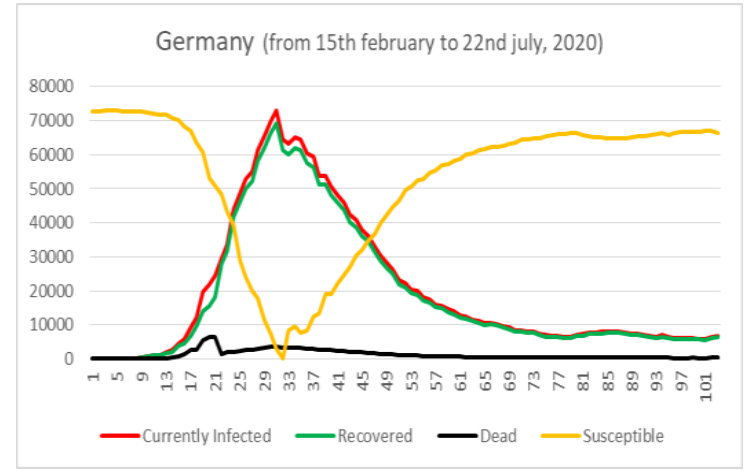

(c)

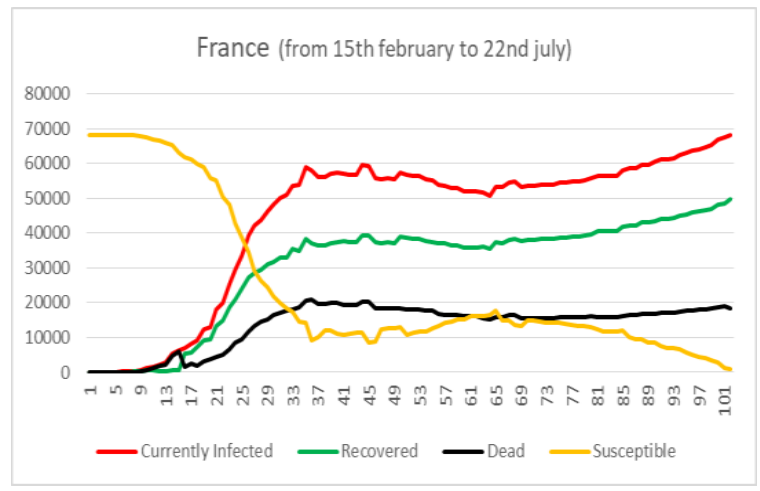

(e)

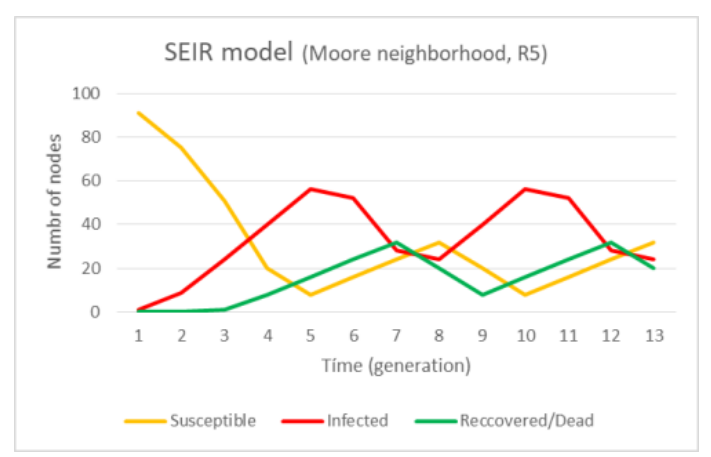

(b)

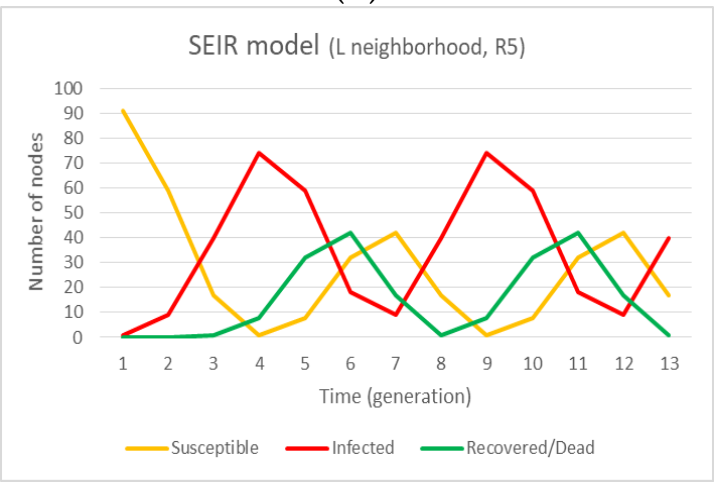

(d)

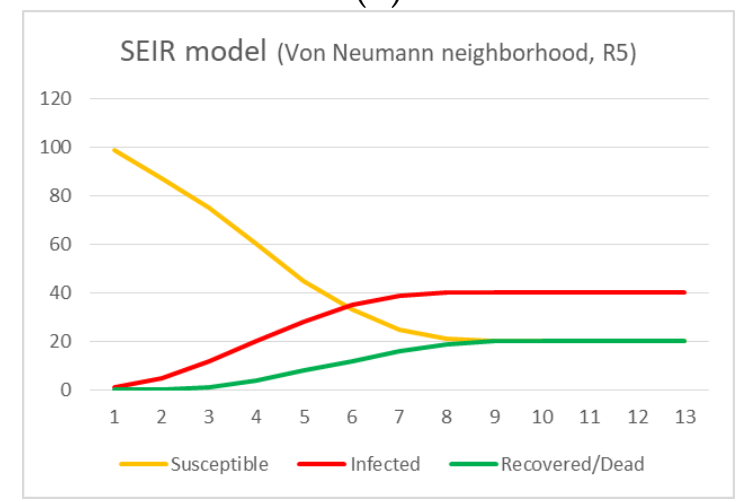

$(\mathbf{f})$

Figure 3. The situation of Covid-19 spread in Italy, Germany and France (a,c,e). The simulation of Susceptible, Currently Infected, Recovered/Dead by means of a Susceptible-Exposed-Infected-Recovered/Dead (SEIR) model, (b,d,f).

In this section we apply our single grid model in order to model the expansion of Covid-19. The suitable paradigm is the SEIR (Susceptible-Exposed-Infected-Recovered/ Dead) since we know the disease has four stages. In Figures 4-6 we present the detailed expansion schedule for the three first generations for the Von Neumann, Moore and L neighbourhoods. The number of Exposed, Infected and Recovered/Dead is then plotted in the graphs shown in Figure $3 \mathrm{~b}, \mathrm{~d}, \mathrm{f}$, respectively, to make easier the comparison between real data and computational approximation. 
Von Neumann neighbourhood, $\mathrm{R}_{5}$

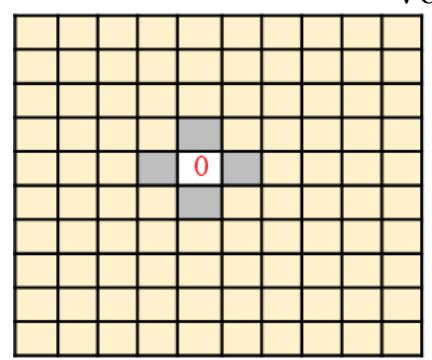

Generation 0:

1 Infected (central red)

4 Exposed (grey)

95 Susceptible (yellow)

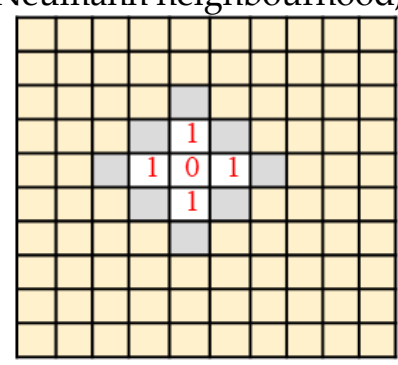

Generation 1:

1 Infected

4 New Infected (red)

8 Exposed (grey)

87 Susceptible (yellow)

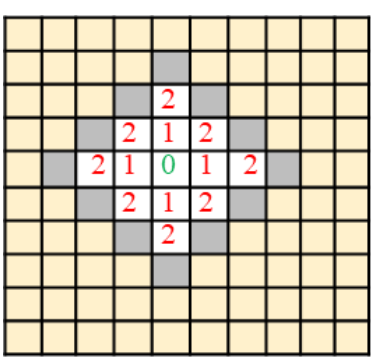

Generation 2:

4 Infected (red)

8 New Infected (red)

1 recovered/dead (green)

12 Exposed (grey)

75 Susceptible (yellow)

Figure 4. Expansion schedule shown for the three first generations (Von Neumann neighbourhood).

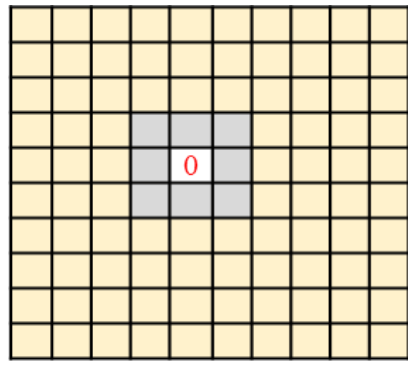

Generation 0:

1 Infected (red)

8 Exposed (grey)

91 Susceptible (yellow)

Moore neighbourhood, $\mathrm{R}_{5}$

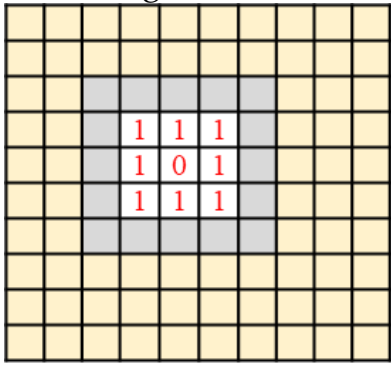

Generation 1:

1 Infected (red)

8 New Infected (red)

16 Exposed (grey)

75 Susceptible (yellow)

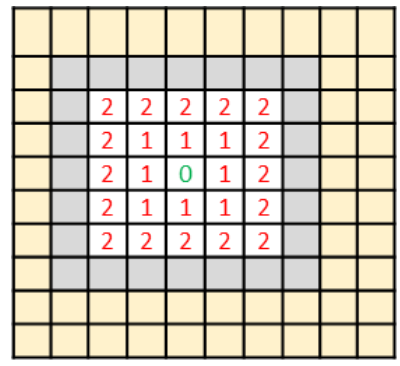

Generation 2:

8 Infected (red)

16 New Infected (red)

1 Recovered

24 Exposed (grey) 51 Susceptible (yellow)

Figure 5. Expansion schedule shown for the three first generations (Moore neighbourhood).

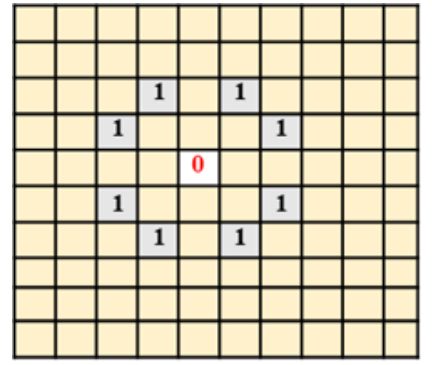

Generation 0:

1 Infected (red)

8 Exposed (grey)

91 Susceptible (yellow)
L neighbourhood, $\mathrm{R}_{5}$

\begin{tabular}{|l|l|l|l|l|l|l|l|l|l|}
\hline & & 2 & & 2 & & 2 & & & \\
\hline & 2 & & 2 & & 2 & & 2 & & \\
\hline 2 & & & 1 & 2 & 1 & & & 2 & \\
\hline & 2 & 1 & 2 & & 2 & 1 & 2 & & \\
\hline 2 & & 2 & & 0 & & 2 & & 2 & \\
\hline & 2 & 1 & 2 & & 2 & 1 & 2 & & \\
\hline 2 & & & 1 & 2 & 1 & & & 2 & \\
\hline & 2 & & 2 & & 2 & & 2 & & \\
\hline & & 2 & & 2 & & 2 & & & \\
\hline & & & & & & & & & \\
\hline
\end{tabular}

Generation 1:

1 Infected (red)

8 New Infected (red)

32 Exposed (grey)

59 Susceptible (yellow)

\begin{tabular}{|l|l|l|l|l|l|l|l|l|l|}
\hline & 3 & 2 & 3 & 2 & 3 & 2 & 3 & & 3 \\
\hline 3 & 2 & 3 & 2 & 3 & 2 & 3 & 2 & 3 & \\
\hline 2 & 3 & & 1 & 2 & 1 & & 3 & 2 & 3 \\
\hline 3 & 2 & 1 & 2 & 3 & 2 & 1 & 2 & 3 & \\
\hline 2 & 3 & 2 & 3 & 0 & 3 & 2 & 3 & 2 & 3 \\
\hline 3 & 2 & 1 & 2 & 3 & 2 & 1 & 2 & 3 & \\
\hline 2 & 3 & & 1 & 2 & 1 & & 3 & 2 & 3 \\
\hline 3 & 2 & 3 & 2 & 3 & 2 & 3 & 2 & 3 & \\
\hline & 3 & 2 & 3 & 2 & 3 & 2 & 3 & & 3 \\
\hline 3 & & 3 & & 3 & & 3 & & 3 & \\
\hline
\end{tabular}

Generation 2:

8 Infected (red)

32 New Infected (red)

1 Recovered

42 Exposed (grey)

17 Susceptible (yellow)

Figure 6. Expansion schedule shown for the three first generations (L neighbourhood). 
In the examples shown in Figures $4-6$, the rule $R_{5}$ was chosen because we are interested in modelling the following situations:

- $\quad$ infection: an infected cell (1) infects a susceptible cell (0) which updates its value to (1), that is to say $(1 * 0)=1$

- healing: an infected cell (1) can spontaneously recover and update to (0), after a determined number of generations

- $\quad$ a susceptible cell has no effect on another susceptible cell so, $(0 * 0)=0$

- $\quad$ an infected cell has no effect on another infected cell so, $(1 * 1)=1$

We do not care about the result of $(0 * 1)$, because this situation does not arise.

We highlight that this situation can be simulated by $\mathrm{R}_{5}$, but it is not the only solution; $R_{7}$ could be suitable too, but not $R_{3}$.

\subsection{Discussion}

In the following, we compare the results of our model with the real Covid-19 data.

- Italy (Figure 3a,b):

In the case of Italy, the pandemic reaches its first peak at $t=40$, corresponding to April 20th with 108,047 currently infected people and 67\% recovered. At the same time, the number of Susceptibles decreases and increases again, due to new infections and/or reinfections of the cured people. The peak of Recovered is achieved at $t=45$, corresponding to April 27th with 74,955 people (71\%). The higher rate of Deaths is achieved between April 5th and 17th (35-42\%). As for Recovered and Infected, the peak is quite flat.

Our best approximation is carried our by the Moore neighborhood and update rule $R_{5}$. The trend is the same: slow increase and decrease, adequate delay between Infected and Recovered. In this case, the generation may be equivalent to little more than a week (10-12 days). However, the height of the peak should be higher for optimal fit. The matching may be improved by means of a multi-grid approach to correct the mismatch. The computational model runs in two cycles. For our comparison, we only consider the first cycle.

\section{- $\quad$ Germany (Figure 3c,d):}

In the case of Germany, the pandemic reaches its first peak at $t=31$, corresponding to April 6th, with 72,865 currently infected people and $95 \%$ recovered. At the same time, the number of Susceptibles decreases and increases again, due to new infections and/or reinfections of yet recovered people. We observe an almost perfect match between Infected and Recovered, this is due to the very high healing rate. Obviously, the death rate is low (only in March was the rate $25 \%$, for the rest of the time it is about $5 \%$ ). This is a very fast spread (2 months, 17th March-13th June).

Our best approximation is carried out by the $\mathrm{L}$ neighborhood and update rule $\mathrm{R}_{5}$. The trend is the same: steep increase and decrease, fast spread. In this case, the generation may be little more than a week (10-12 days). Nevertheless, we do not have a perfect matching between the Infected and Recovered values. To produce a better result, we could model this case with a two-grid implementation to correct the mismatch. The computational model runs in two cycles. For our comparison, we only consider the first cycle.

- $\quad$ France (Figure 3e,f):

In the case of France, the pandemic reaches its highest value of currently infected people at $\mathrm{t}=36$, which corresponds to 14 th April with 38,384 people. At the same time, the number of Susceptibles decreases and decreases again after a constant stage. This is due to the similar behavior of Recovered or Dead, that achieve an equilibrium value about $68-70 \%$ and $30-32 \%$, respectively (the first is two times the second). The evolution of Infected, Recovered and Dead is very parallel. This outbreak is speedy in the increase but we do not observe any decrease. It seems to be a persistent pandemic.

Our best approximation is achieved by the Von Neumann neighborhood and update rule $R_{5}$. This scheme shows very clearly the parallel evolution of the pandemic data for 
Infected and Recovered from generation $\mathrm{n}^{\circ} 7$ with an equilibrium value of 40 and $20 \%$ (the first is also two times the second). The generation corresponds to 6 days, more or less. The worst result is for the level of the Infected, which is too low. This drawback can be overcome by using a two-grid implementation.

We conclude that our model is a valuable tool to provide a first qualitative approximation to the pandemic expansion. The actual trends are correctly depicted by our simulation. A more detailed match can be achived by a multi-grid implementation that is successful in correcting for bias. This second part of the model falls out of the scope of the present paper.

\section{Conclusions}

The fight against Covid-19 has taught us a lot in a short time. In addition to the importance of knowing the virus and having treatment and vaccines, it is crucial to have a correct management of the pandemic because it has become clear that the main drawbacks of outbreak management came from the pressure in ICUs and hospitals that were on the verge of collapse. The management of the pandemic with the modification of its parameters can be an alternative way to avoid the overflow of the sanitary structures. Forecasting is achieved if we are able to find the keys to expansion in order to control it. In this sense, our basic model succeeds in pointing out some parameters that are crucial to the expansion phenomena, such as neighborhoods, update rules and delays. This approach provides a valuable tool to deal with the expansion phenomena. The single grid model plays the role of a template, which provides a correct shape (behavior) for the expansion process. A more precise approximation can be achieved using the multi-grid approach. In the three presented cases, one more grid may be suitable to raise the levels of Infected for Italy and France, and to correct the delay between Infected and Recovered for Germany.

As future work, we plan to improve the model. We need to develop criteria to estimate whether or not an approximation is fine enough in order to decide iteratively to add another grid or not. Moreover, the multi-grid application may be useful to understand the contribution of different parameters in the dynamics of a given phenomenon. This could provide a method to discard the less important parameters in the control policies and focus attention on the most important ones. The fight against pandemic and against plant pests are the fields in which we intend to apply the method.

Author Contributions: Conceptualization, M.T.S.-P. and H.M.-M.; methodology, M.T.S.-P. and J.J.C.P.; software, J.J.C.-P., validation, M.T.S.-P. and H.M.-M.; formal analysis, H.M.-M. and J.J.C.-P.; investigation, M.T.S.-P., H.M.-M. and J.J.C.-P.; resources, H.M.-M.; data curation, J.J.C.-P.; writingoriginal draft preparation, H.M.-M.; writing—review and editing, M.T.S.-P.; visualization, M.T.S.-P.; supervision, M.T.S.-P.; project administration, M.T.S.-P. and H.M.-M. All authors have read and agreed to the published version of the manuscript.

Funding: This research received no external funding.

Conflicts of Interest: The authors declare no conflict of interest.

\section{References}

1. Abou-Ismail, A. Compartmental Models of the COVID-19 Pandemic for Physicians and Physician-Scientists. SN Compr. Clin. Med. 2020, 2, 852-858. [CrossRef] [PubMed]

2. Fosu, G.O.; Opong, J.M.; Appati, J.K. Construction of Compartmental Models for COVID-19 with Quarantine, Lockdown and Vaccine Interventions. SSRN Electron. J. 2020. [CrossRef]

3. Wang, T.; Wu, Y.; Lau, J.Y.-N.; Yu, Y.; Liu, L.; Li, J.; Zhang, K.; Tong, W.; Jiang, B. A four-compartment model for the COVID-19 infection-Implications on infection kinetics, control measures, and lockdown exit strategies. Precis. Clin. Med. 2020, 3, 104-112. [CrossRef]

4. Tang, L.; Zhou, Y.; Wang, L.; Purkayastha, S.; Zhang, L.; He, J.; Wang, F.; Song, P.X. A Review of Multi-Compartment Infectious Disease Models. Int. Stat. Rev. 2020, 88, 462-513. [CrossRef] [PubMed]

5. Arino, J.; Portet, S. A simple model for COVID-19. Infect. Dis. Model. 2020, 5, 309-315. [CrossRef] [PubMed]

6. Serhani, M.; Labbardi, H. Mathematical modeling of COVID-19 spreading with asymptomatic infected and interacting peoples. J. Appl. Math. Comput. 2020, 17, 1-20. [CrossRef] [PubMed] 
7. Bordehore, C.; Navarro, M.; Herrador, Z.; Fonfria, E.S. Understanding COVID-19 Spreading through Simulation Modeling and Scenarios Comparison: Preliminary Results. Available online: https://www.medrxiv.org/content/10.1101/2020.03.30.2004704 3v1.full.pdf (accessed on 5 February 2021).

8. Burgos-Simón, C.; Cortés, J.C.; Lopez-Navarro, E.; Martinez-Rodriguez, D.; Martinez-Rodriguez, P.; Julián, R.S.; Villanueva, R.J. Modelo Para el Estudio de la Dinámica de Transmisión del SARS-COV-2 y la Enfermedad del COVID19. Descripción Técnica. Instituto Universitario de Matemática Multidisciplinar, Universitat Politècnica de Valencia, 46022. Available online: https:/ / covid19.webs.upv.es/ (accessed on 5 February 2021).

9. Signes-Pont, M.T.; Mora, H.M.; Cortés, A. The Susceptible-Infectious Model of disease expansion analysed under the scope of connectivity and neighbour rules. Comput. Sci. Inf. Technol. 2017, 7, 1-10.

10. Signes-Pont, M.T.; Mora, H.M.; Castillo, A.C. A discrete approach of the Susceptible-Infectious-Susceptible (SIS) Model of Disease Expansion. Int. J. Comput. 2017, 2, 123-128.

11. Signes-Pont, M.T.; Mora, H.M.; Castillo, A.C. The Susceptible-Infectious-Recovered (SIR) model of disease expansion: A new approach. In Proceedings of the 17th Edition of the Mathematical Modelling in Engineering and Human Behaviour Conference, Valencia, Spain, 8-10 July 2020.

12. Signes-Pont, M.T.; Ramirez-Martinez, D.E.; García-Chamizo, J.M.; Mora, H. A multigrid approximation to the expansion of Xylella fastidiosa in almond trees. WSEAS Trans. Comput. 2019, 18, 19-30.

13. Signes-Pont, M.T.; Cortés-Plana, J.J.; Mora, H.; Mollá-Sirvent, R. An epidemic model to address the spread of plant pests. The case of Xylella fastidiosa in almond trees. Kybernetes 2020. [CrossRef]

14. Signes-Pont, M.T.; Mora, H.; Cortés-Castillo, A.; Mollá-Sirvent, R.A. A grid model to address the behaviour of the nodes in the dissemination of information. Kybernetes 2019, 49, 1953-1967. [CrossRef]

15. Signes-Pont, M.T.; Mora-Mora, H.; Mollá-Sirvent, R.; Cortés-Castillo, A. A framework to address the impact of people's behavior in the dissemination of information through mobile social networks. In Proceedings of the 6th Business Systems Laboratory International Symposium, Pavía, Italia, 21-23 January 2019.

16. Cortés-Castillo, A. Modelización de la Propagación de Información a Través de las Redes Informáticas. Ph.D. Thesis, University of Alicante, Alicante, Spain, July 2020.

17. COVID-19 Outbreak Live Update. Available online: https://www.worldometers.info/coronavirus/ (accessed on 14 March 2020). 